\title{
Precarização do mercado de trabalho de auxiliares e técnicos de Enfermagem no Ceará, Brasil
}

\author{
Labor market insecurity for nursing assistants and technicians \\ in the State of Ceará, Brazil
}

Raimunda Magalhães da Silva (https://orcid.org/0000-0001-5353-7520) ${ }^{1}$

Luiza Jane Eyre de Souza Vieira (https://orcid.org/0000-0002-5220-027X) ${ }^{1}$

Carlos Garcia Filho (https://orcid.org/0000-0002-0345-6033) ${ }^{1}$

Indara Cavalcante Bezerra (https://orcid.org/0000-0003-0647-2490) ${ }^{1}$

Ana Neiline Cavalcante (https://orcid.org/0000-0002-6787-7216) ${ }^{1}$

Fernanda Colares de Borba Netto (https://orcid.org/0000-0002-2621-1135) ${ }^{1}$

Francisca Alanny Rocha Aguiar (https://orcid.org/0000-0002-6281-4523) ${ }^{1}$
${ }^{1}$ Programa de Pós-

Graduação em Saúde

Coletiva, Universidade de

Fortaleza. Av. Washington

Soares 1321, Edson Queiroz.

60811-905 Fortaleza CE

Brasil.rmsilva@unifor.br

\begin{abstract}
The article analyzes the labor market of nursing assistants and technicians in the State of Ceará, Brazil, from 2013 to 2017, concerning job insecurity aspects. Two data sources were employed. The cross-sectional Brazilian Nursing Team Profile Survey (PPEB) was used as the baseline, and the longitudinal Annual List of Social Information (RAIS) database, allowed the study of the trend of some of the variables related to job insecurity. Given the small number of similar studies for this professional category, we opted for an exploratory data approach that supported a critical discussion of the results. The job insecurity of nursing assistants and technicians is supported by empirical evidence in the four perspectives adopted by the International Labor Organization-ILO: time-related, economic, social, and organizational. While it is a market with a positive balance of admissions compared to layoffs, jobs have low salaries, poor work relationships, high turnover, lack of prospect of career advancement, and adverse working conditions such as exposure to violence, discrimination, and accidents. We can conclude that RAIS data corroborate the PPEB findings, and point out that there is no trend of improvement for this situation.
\end{abstract}

Key words Employment, Job market, Nursing
Resumo O artigo analisa o mercado de trabalho de auxiliares e técnicos de enfermagem no Ceará, nos anos de 2013 a 2017, nos aspectos relacionados à precarização do trabalho. Duas fontes de dados foram empregadas. A Pesquisa sobre o Perfil da Equipe de Enfermagem no Brasil (PPEB), de caráter transversal, foi utilizada como linha de base, e a Relação Anual de Informações Sociais-RAIS, longitudinal, possibilitou o estudo da evolução de algumas das variáveis relacionadas à precarização do trabalho. Dada a pequena quantidade de estudos semelhantes para essa categoria profissional, optou-se por realizar uma abordagem exploratória que subsidiou uma discussão crítica dos resultados. A precarização do trabalho de auxiliares e técnicos de enfermagem é sustentada por evidências empíricas nas quatro perspectivas adotadas pela Organização Internacional do Trabalho-OIT: temporal, econômica, social e organizacional. Embora seja um mercado com saldo positivo de admissões em relação aos desligamentos, os empregos apresentam baixos salários, vínculos precários, alta rotatividade, ausência de perspectiva de ascensão e condições adversas de trabalho, como exposição a violência, discriminação e acidentes. Conclui-se que os dados da RAIS corroboram os achados da PPEB e apontam que inexiste qualquer tendência de melhora dessa situação.

Palavras-chave Emprego, Mercado de trabalho, Enfermagem 


\section{Introdução}

É provável que o primeiro contato da população no serviço de saúde seja com a equipe de Enfermagem, que representa metade da força de trabalho em saúde no Brasil e agrega mais de 2 milhões de trabalhadores nos níveis auxiliar, técnico e superior, formada, aproximadamente, de $23 \%$ de enfermeiros e $77 \%$ de técnicos e auxiliares de enfermagem ${ }^{1-4}$.

Os auxiliares e técnicos de Enfermagem atuam ao lado de enfermeiros, perfazendo uma força de trabalho que vem aumentando nos últimos anos, pela carência de enfermeiros, contenção de custos, mudanças na prática do enfermeiro quanto as habilidades técnicas, funções especializadas e burocracia nos processos de trabalho ${ }^{5}$.

As designações dos auxiliares e técnicos variam entre países. Na Inglaterra, pode ser chamado de assistente de saúde, trabalhador de apoio de saúde ou auxiliar de enfermagem; na Austrália, assistente de atendimento; nos Estados Unidos, auxiliar de enfermagem certificado ou pessoal de assistência não licenciado, a depender do registro ${ }^{5}$. No Brasil, as designações variam com o grau de instrução; o Auxiliar deve ter o ensino fundamental completo e habilitação em curso de Auxiliar de Enfermagem registrado no Conselho Regional de Enfermagem (COREN) e o Técnico de Enfermagem, nível médio completo e curso de Técnico de Enfermagem com registro no COREN $^{6}$.

A despeito da quantidade significativa de profissionais nos municípios, inserida no Sistema Único de Saúde (SUS) e atuante nos setores público, privado, filantrópico e de ensino, a força de trabalho da Enfermagem ainda é pouco estudada. Por essa razão, a pesquisa 'Perfil da Enfermagem no Brasil' (PPEB), iniciada em 2013, traçou o perfil dessa população, com a construção de amplo banco de dados para diagnosticar e debater a situação dos enfermeiros, técnicos e auxiliares de Enfermagem em atuação no Brasil ${ }^{7}$. Até este momento, seus resultados evidenciaram características desses profissionais e destaca-se a distribuição desigual no território nacional, concentrada na região Sudeste e nas capitais com predomínio da força de trabalho feminina com salários baixos e jornadas de trabalho elevadas ${ }^{1-4}$.

Investigações discorrem sobre esse contingente contemplando diferentes objetos de estudo, como a sistematização do processo de trabalho em Enfermagem ${ }^{8-12}$ e grau de satisfação e/ou insatisfação profissional ${ }^{13}$. Identificaram dimen- sões que circunscrevem a precarização no trabalho, como a estabilidade de vínculo, remuneração, carga horária e segurança no trabalho ${ }^{14-17}$, no entanto sem explorar tópicos que impliquem em estratégias resolutivas à melhoria da qualidade de vida desses profissionais, especialmente técnicos e auxiliares, e que podem comprometer a saúde, além da segurança do paciente.

A Enfermagem, como outras profissões da saúde, enfrenta degradação das relações de trabalho, baixos salários e condições de trabalho inadequadas. Os vínculos empregatícios instáveis são comuns, o que denota fragilidade, perda de direitos trabalhistas, resultando em desânimo, excesso de desgaste e baixa qualidade da assistência prestada ${ }^{16-18}$.

Ainda que inexista consenso sobre a definição de precarização do trabalho na literatura, para a Organização Internacional do Trabalho-OIT ${ }^{19}$, algumas dimensões balizam a compreensão: a dimensão temporal, que reporta-se a incerteza sobre a manutenção do emprego; a organizacional, ausência de controle individual ou coletivo sobre as condições de trabalho; a econômica, caracterizada por baixos salários e ausência de perspectiva de aumentos; e a social que refere-se a proteção deficiente contra demissão injusta, discriminação, práticas de trabalho inaceitáveis e ausência de benefícios da seguridade social.

No Ceará, estado do Nordeste brasileiro, a PPEB abrangeu 42.462 técnicos e auxiliares de enfermagem e apontou a existência de importantes assimetrias nas condições de trabalho nas quais essa categoria profissional exerce suas atividades ${ }^{7}$. A despeito do Ceará destacar-se como um dos pioneiros na adesão e incorporação de políticas e programas para ampliar o acesso da população aos serviços de saúde e alcançar melhoria nos seus indicadores, perpetuam-se as iniquidades sociais ${ }^{20}$.

A sobreposição desses eventos - existência de assimetrias no interior da categoria profissional e de assimetrias no contexto social mais amplo possivelmente coloca o Estado do Ceará em condição profícua ao agravamento da precarização do trabalho, considerado pela OIT $^{19}$ como uma tendência global.

Nessa lógica, este artigo propõe-se a analisar o mercado de trabalho de auxiliares e técnicos de enfermagem no Ceará, no período de 2013 a 2017, com ênfase nos aspectos relacionados à precarização do trabalho dessa categoria, nas dimensões temporal, econômica, social e organizacional. 


\section{Método}

Essa pesquisa utilizou duas fontes de dados. A Pesquisa sobre o Perfil da Equipe de Enfermagem no Brasil $(\mathrm{PPEB})^{7}$, de caráter transversal, e a Relação Anual de Informações Sociais (RAIS) ${ }^{21}$ dos anos de 2013 a 2017. Não foi utilizado o ano de 2018 da RAIS, pois não estava disponível para acesso público durante a elaboração deste artigo.

A PPEB teve como objetivo analisar a situação atual da enfermagem no país em vários aspectos, buscando conhecer a sua dinâmica no contexto socioeconômico e político brasileiro. Sua população abrangeu os enfermeiros, técnicos e auxiliares de enfermagem do Brasil, que possuíam, na épo$\mathrm{Ca}$, registro ativo no COFEN. A PPEB tem representatividade nacional, capaz de gerar resultados para cada unidade da federação. Sua amostragem foi aleatória e o instrumento de coleta um questionário postado para todos os respondentes ${ }^{7}$.

A Relação Anual de Informações Sociais (RAIS) é um registro administrativo nacional e obrigatório para todos os estabelecimentos, inclusive aqueles sem ocorrência de vínculo empregatício. A RAIS é elaborada pelo Ministério da Economia e objetiva prover informações para o controle da atividade trabalhista no País, subsidiar a elaboração de estatísticas do trabalho e disponibilizar informações do mercado de trabalho às entidades governamentais. As Bases de Dados da RAIS são geradas a partir de análise das declarações originais preenchidas pelos estabelecimentos. Após organização e checagem dos dados, os bancos de dados são disponibilizados para acesso público. Quanto à unidade de análise, as informações dos empregados são prestadas para cada vínculo de emprego, portanto um trabalhador pode constar na RAIS mais de uma vez, de acordo com a quantidade de vínculos que possui no ano da coleta ${ }^{21}$.

Utilizou-se a linguagem R e o software R-Studio versão 1.1.456 para manusear os bancos de dados da RAIS e o Microsoft Excel para elaborar os gráficos e as Tabelas. Realizou-se uma abordagem descritiva dos dados comparando, sempre que possível, as duas fontes. A PPBE foi utilizada como linha de base transversal e a RAIS como possibilidade de estudo longitudinal de algumas das principais variáveis relacionadas à precarização do trabalho dos técnicos e auxiliares de enfermagem. Considerando a escassez de estudos semelhantes para essa categoria profissional, optou-se por realizar uma abordagem exploratória dos dados que subsidiou uma discussão crítica dos resultados.

\section{Resultados}

Os resultados são apresentados nas dimensões temporal, econômica, social e organizacional, orientadas pela definição de precarização do trabalho pela OIT ${ }^{19}$.

\section{Dimensão temporal}

A PPEB apresenta um quadro positivo da situação funcional dos auxiliares e técnicos de enfermagem. Em 2013, 4,1\% referem estar desempregados, situação próxima do pleno emprego. Sobre a ocorrência de desemprego nos últimos 12 meses, considerando como base o ano de 2013, a PPEB aponta que foi de $8,3 \%$.

Dos profissionais desempregados, $65,9 \%$ tiveram dificuldade de encontrar emprego e 31,7\% responderam negativamente a essa pergunta. Os dois principais motivos apontados para a dificuldade de encontrar emprego foram a "falta de experiência profissional" $(24,2 \%)$ e a "falta de concursos públicos" (21\%), denotando dificuldade de ingresso dos profissionais recém-formados no mercado de trabalho. A PPEB confirma essa situação ao apontar que $67,8 \%$ dos profissionais desempenham atividades no setor público.

A quantidade de atividades realizadas pelos entrevistados na PPEB supera (57.070) o contingente de entrevistados (42.016). A média de atividades por profissional é de 1,35. As atividades mais frequentes são realizadas no setor público estadual $(27,6 \%)$, municipal $(21,5 \%)$ e cooperativa $(18,2 \%)$, terceira mais comum, superando, inclusive, a atividade no setor privado (15,8\%). Por outro lado, mais da metade das atividades realizadas são no setor público $(51,2 \%)$, que de modo geral é visto como emprego de estabilidade, dada a identificação dessas atividades com a condição de estatutário.

O predomínio de atividades no setor público deveria, a priori, indicar que a categoria profissional não sofre com instabilidade de vínculo, uma das características do trabalho precário. Contudo, quando se observa os tipos de vínculos em cada uma das atividades (Tabela 1), constatase uma situação diferente do esperado, pois, no setor público, o vínculo por meio de cooperativa $(30,7 \%)$ rivaliza com o estatutário $(35,7 \%)$. Os contratos por tempo determinado e a prestação de serviços sem vínculo constituem mais da metade dos vínculos de trabalho. No setor privado e no filantrópico, a proporção de vínculo celetista é de $62,1 \%$ e $52,4 \%$, que pode ser considerada até melhor que no setor público, tendo em vista a 
Tabela 1. Auxiliares e Técnicos de Enfermagem segundo tipo vínculo empregatício por setor de atuação em 2013.

\begin{tabular}{lcccccccccc}
\hline \multirow{2}{*}{ Vínculos } & \multicolumn{2}{c}{ Público } & \multicolumn{2}{c}{ Privado } & \multicolumn{2}{c}{ Filantrópico } & \multicolumn{2}{c}{ Ensino } & \multicolumn{2}{c}{ Total } \\
\cline { 2 - 12 } & $\mathbf{n}$ & $\%$ & $\mathbf{n}$ & $\%$ & $\mathbf{n}$ & $\%$ & $\mathbf{n}$ & $\%$ & $\mathbf{n}$ & $\%$ \\
\hline Estatutário & 10.802 & 35,2 & 0 & 0,0 & 0 & 0,0 & 680 & 66,7 & 11.482 & 26,5 \\
Celetista & 2.296 & 7,5 & 5.018 & 62,1 & 1.871 & 52,4 & 170 & 16,7 & 9.355 & 21,6 \\
Temporário & 1.191 & 3,9 & 510 & 6,3 & 595 & 16,7 & 85 & 8,3 & 2.381 & 5,5 \\
P. Serviço* & 3.742 & 12,2 & 1.276 & 15,8 & 851 & 23,8 & - & - & 5.869 & 13,5 \\
Cooperativado & 9.441 & 30,7 & 1.021 & 12,6 & 170 & 4,8 & - & - & 10.632 & 24,5 \\
Fundação Privada & 510 & 1,7 & - & - & - & - & - & - & 510 & 1,2 \\
FPDP** & 1.190 & 3,9 & - & - & - & - & - & - & 1.190 & 2,7 \\
OSCIP*** & 85 & 0,3 & - & - & - & - & - & - & 85 & 0,2 \\
OS ${ }^{* * *}$ & 85 & 0,3 & - & - & - & - & - & - & 85 & 0,2 \\
Sócio & - & - & - & - & 85 & 2,4 & - & - & 85 & 0,2 \\
Proprietário & - & - & 85 & 1,1 & - & - & - & - & 85 & 0,2 \\
Por conta própria & - & - & 85 & 1,1 & - & - & - & - & 85 & 0,2 \\
Horista & - & - & - & - & - & - & 85 & 8,3 & 85 & 0,2 \\
Outros & 1.361 & 4,4 & 85 & 1,1 & - & - & - & - & 1.446 & 3,3 \\
Total & 30.703 & 100,0 & 8.080 & 100,0 & 3.572 & 100,0 & 1.020 & 100,0 & 43.375 & 100,0 \\
\hline
\end{tabular}

Legenda: P. Serviço* $=$ Prestador de Serviço. FPDP ${ }^{\star *}=$ Fundação Pública de Direito Privado. OSCIP*** = Organização da

Sociedade Civil de Interesse Público. OS ${ }^{\star * *}=$ Organização Social.

Obs.: (Admite-se mais de uma resposta para cada entrevistado)

Fonte: Elaborado a partir da Pesquisa Perfil da Enfermagem no Brasil7.

proteção ao trabalhador fornecida pela CLT em comparação com vínculos mais precários. Considerando a categoria como um todo, $48,1 \%$ dos vínculos são estatutários ou celetistas, sendo os demais por meio de formas alternativas, dentre os quais, o vínculo por meio de cooperativas com $24,5 \%$ e a prestação de serviços com $13,5 \%$.

A frequência de mudança de emprego na categoria nos dois últimos anos, com base em 2013, foi de $11,9 \%$ para uma mudança, $3,4 \%$ para duas e 1,2\% para três ou mais. Os motivos foram a necessidade de conciliar dois ou mais empregos $(19,0 \%)$, insatisfação salarial $(19,0 \%)$ e insatisfação com as condições de trabalho $(12,7 \%)$. As causas apontadas pelos profissionais referem-se a problemas relacionados com a precarização do trabalho da categoria.

A quantidade total de horas trabalhadas por semana, obtida por meio da PPEB, esclarece o porquê da necessidade de conciliar dois ou mais empregos ser um motivo tão relevante para as mudanças. Quase metade dos profissionais $(46,6 \%)$ trabalha mais de 40 horas por semana, carga horária padrão do setor público. Observa-se, também, que $13,8 \%$ trabalha mais que 60 horas/semana. Esse dado legitima o número de atividades maior que a quantidade de profissionais. Por outro lado, observa-se que há poucos profissionais $(1,2 \%)$ que trabalham 20 horas ou menos por semana.
A dinâmica do mercado de trabalho pode ser estudada pelo saldo anual de admissões e desligamentos. O Gráfico 1 mostra saldo positivo para todos os anos estudados, logo, trata-se de um mercado de trabalho em expansão. Contudo também se observa que o saldo tende a diminuir ao longo do tempo. Destaca-se que a menor diferença entre admissões e demissões foi em 2016, quando agravou-se a crise política, econômica e institucional no país.

Outra informação sobre a dinâmica do mercado de trabalho é a rotatividade, que pode ser acessada de modo indireto na RAIS por meio do tempo no emprego (Tabela 2). É possível agrupar os vínculos em três grupos: cerca de 1/4 dos trabalhadores estão no primeiro ano de emprego, $1 / 2$ entre um e cinco anos e os demais com mais de cinco anos. A partir dessa estabilidade entre os grupos, pode-se sugerir que não existe migração dos trabalhadores ingressantes no mercado recentemente para vínculos com perspectiva de maior estabilidade. A queixa sobre os raros concursos públicos feita na PPEB pode corroborar essa hipótese.

\section{Dimensão econômica}

A renda de auxiliares e técnicos de enfermagem sinaliza a precarização do trabalho dessa categoria. A Tabela 3 apresenta os dados do ren- 


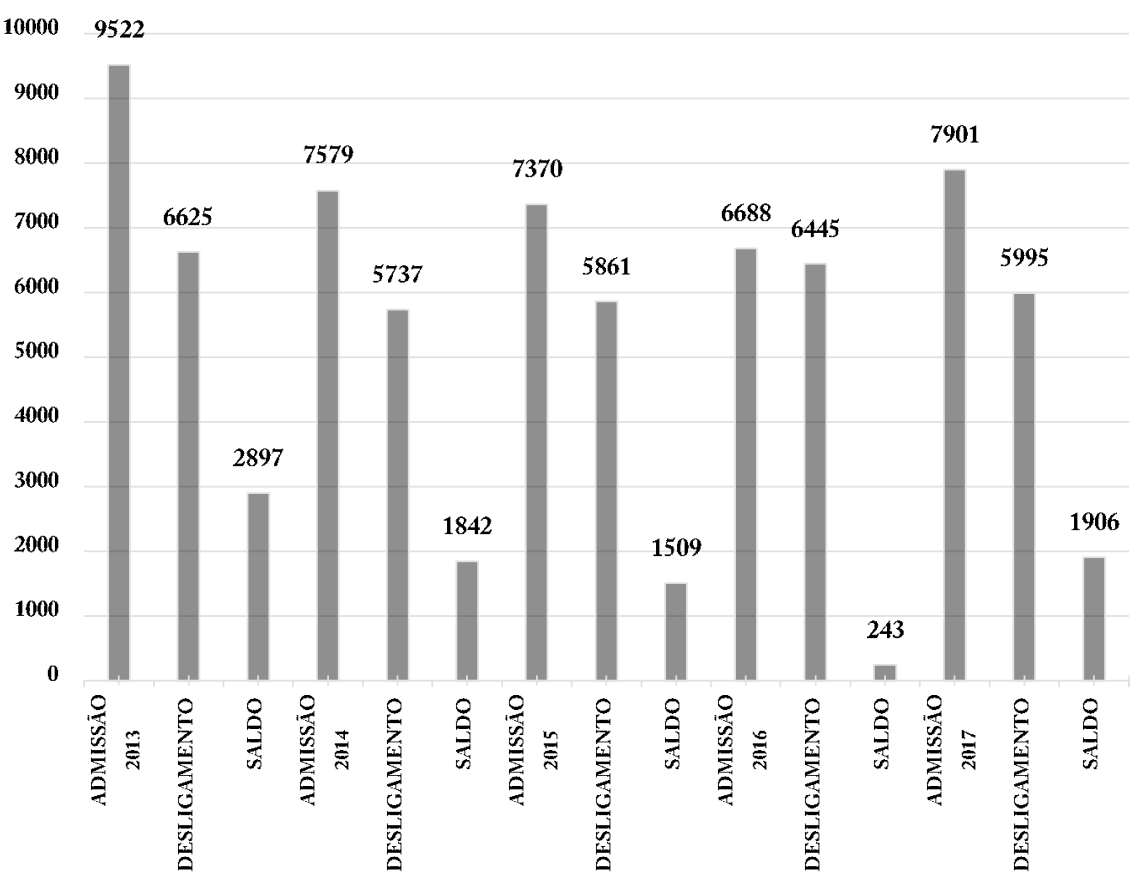

Gráfico 1. Saldo de admissões e desligamentos por ano de 2013 a 2017.

Fonte: Elaborado a partir da RAIS-MTE-2018.

Tabela 2. Tempo no emprego de Auxiliares e Técnicos de Enfermagem em 2013.

\begin{tabular}{|c|c|c|c|c|c|c|c|c|c|c|}
\hline \multirow{2}{*}{$\begin{array}{l}\text { Meses no } \\
\text { emprego }\end{array}$} & \multicolumn{2}{|c|}{2013} & \multicolumn{2}{|c|}{2014} & \multicolumn{2}{|c|}{2015} & \multicolumn{2}{|c|}{2016} & \multicolumn{2}{|c|}{2017} \\
\hline & V.Abs. & $\%$ & V.Abs. & $\%$ & V.Abs. & $\%$ & V.Abs. & $\%$ & V.Abs. & $\%$ \\
\hline até 2,9 & 2.529 & 8,41 & 2.222 & 7,38 & 2.443 & 7,90 & 1.928 & 6,13 & 2.224 & 6,89 \\
\hline 3,0 a 5,9 & 3.185 & 10,60 & 2.184 & 7,25 & 2.056 & 6,65 & 2.209 & 7,02 & 2.231 & 6,91 \\
\hline 6,0 a 11,9 & 4.506 & 14,99 & 3.936 & 13,06 & 3.689 & 11,93 & 3.281 & 10,43 & 4.077 & 12,63 \\
\hline 12,0 a 23,9 & 3.407 & 11,34 & 5.461 & 18,13 & 4.308 & 13,93 & 4.509 & 14,33 & 3.618 & 11,21 \\
\hline 24,0 a 35,9 & 2.499 & 8,32 & 2.253 & 7,48 & 3.798 & 12,28 & 3.027 & 9,62 & 2.835 & 8,78 \\
\hline 36,0 a 59,9 & 2.972 & 9,89 & 3.201 & 10,62 & 3.426 & 11,08 & 4.558 & 14,48 & 4.712 & 14,59 \\
\hline 60,0 a 119,9 & 4.440 & 14,77 & 4.158 & 13,80 & 4.268 & 13,80 & 4.468 & 14,20 & 4.565 & 14,14 \\
\hline 120,0 ou mais & 6.498 & 21,62 & 6.703 & 22,25 & 6.935 & 22,42 & 7.462 & 23,71 & 8.016 & 24,83 \\
\hline Não classificado & 18 & 0,06 & 10 & 0,03 & 9 & 0,03 & 27 & 0,09 & 10 & 0,03 \\
\hline Total & 30.054 & 100,00 & 30.128 & 100,00 & 30.932 & 100,00 & 31.469 & 100,00 & 32.288 & 100,00 \\
\hline
\end{tabular}

Fonte: Elaborado a partir da RAIS-MTE-2018.

dimento mensal total dos profissionais com base no salário mínimo de 2013. A Tabela 4 mostra a evolução da remuneração média por vínculo nos últimos cinco anos.

Os resultados das duas tabelas são convergentes ao apontarem um predomínio de remuneração igual ou pouco superior ao salário mínimo oficial e sem tendência de melhoria. A situação dos trabalhadores do setor público é melhor que a do setor privado, que, por sua vez, supera a do setor filantrópico. $\mathrm{Na}$ PPEB os profissionais foram questionados quanto ao valor ideal de sua remuneração e o resultado mais prevalente foi um valor entre $\mathrm{R} \$ 1.000,00$ até $\mathrm{R} \$ 2.000,00$, ape- 
Tabela 3. Auxiliares e Técnicos de Enfermagem por renda mensal em 2013.

\begin{tabular}{|c|c|c|c|c|c|c|c|c|c|c|c|c|}
\hline \multirow{2}{*}{$\begin{array}{c}\text { Total de } \\
\text { rendimento } \\
\text { (mensal em } \\
\text { reais) }\end{array}$} & \multicolumn{2}{|c|}{ Setor Público } & \multicolumn{2}{|c|}{ Setor Privado } & \multicolumn{2}{|c|}{$\begin{array}{c}\text { Setor } \\
\text { Filantrópico }\end{array}$} & \multicolumn{2}{|c|}{$\begin{array}{l}\text { Setor de } \\
\text { Ensino }\end{array}$} & \multicolumn{2}{|c|}{$\begin{array}{c}\text { Total nas } \\
\text { Atividades de } \\
\text { Enfermagem }\end{array}$} & \multicolumn{2}{|c|}{$\begin{array}{c}\text { Salário } \\
\text { Mensal Ideal }\end{array}$} \\
\hline & V.Abs. & $\%$ & V.Abs. & $\%$ & V.Abs. & $\%$ & V.Abs. & $\%$ & V.Abs. & $\%$ & V.Abs. & $\%$ \\
\hline Menos de $680^{*}$ & 3.487 & 12,2 & 1.786 & 19,8 & 1.531 & 40,1 & 170 & 13,3 & 3.317 & 7,9 & 170 & 0,4 \\
\hline $681-1000$ & 8.760 & 30,7 & 4.253 & 47,2 & 1.276 & 33,4 & 170 & 13,3 & 16.670 & 39,7 & 1.446 & 3,4 \\
\hline $1001-2000$ & 8.931 & 31,3 & 1.021 & 11,3 & 255 & 6,7 & 425 & 33,3 & 11.227 & 26,7 & 12.588 & 30,0 \\
\hline $2001-3000$ & 1.871 & 6,6 & 170 & 1,9 & 0 & 0,0 & 340 & 26,7 & 2.126 & 5,1 & 7.400 & 17,6 \\
\hline $3001-4000$ & 255 & 0,9 & 0 & 0,0 & 0 & 0,0 & 0 & 0,0 & 340 & 0,8 & 2.552 & 6,1 \\
\hline $4001-5000$ & 85 & 0,3 & 0 & 0,0 & 0 & 0,0 & 0 & 0,0 & 170 & 0,4 & 1.701 & 4,0 \\
\hline $5001-6000$ & 0 & 0,0 & 0 & 0,0 & 0 & 0,0 & 0 & 0,0 & 0 & 0,0 & 170 & 0,4 \\
\hline $6001-7000$ & 0 & 0,0 & 0 & 0,0 & 0 & 0,0 & 0 & 0,0 & 170 & 0,4 & 0 & 0,0 \\
\hline $7001-8000$ & 0 & 0,0 & 0 & 0,0 & 0 & 0,0 & 0 & 0,0 & 0 & 0,0 & 0 & 0,0 \\
\hline $8001-9000$ & 0 & 0,0 & 0 & 0,0 & 0 & 0,0 & 0 & 0,0 & 0 & 0,0 & 0 & 0,0 \\
\hline $9001-10000$ & 0 & 0,0 & 0 & 0,0 & 0 & 0,0 & 0 & 0,0 & 0 & 0,0 & 85 & 0,2 \\
\hline $10.001-20.000$ & 0 & 0,0 & 0 & 0,0 & 0 & 0,0 & 0 & 0,0 & 85 & 0,2 & 85 & 0,2 \\
\hline Mais de 20.000 & 0 & 0,0 & 0 & 0,0 & 0 & 0,0 & 0 & 0,0 & 0 & 0,0 & 0 & 0,0 \\
\hline $\begin{array}{l}\text { Não } \\
\text { Respondido }\end{array}$ & 5.103 & 17,9 & 1.786 & 19,8 & 756 & 19,8 & 170 & 13,3 & 7.910 & 18,8 & 15.820 & 37,7 \\
\hline Total & 28.492 & 100,0 & 9.016 & 100,0 & 3.818 & 100,0 & 1275 & 100,0 & 42.015 & 100,0 & 42.017 & 100,0 \\
\hline
\end{tabular}

Tabela 4. Auxiliares e Técnicos de Enfermagem remuneração em salários mínimos por vínculo de 2013 a 2017.

\begin{tabular}{|c|c|c|c|c|c|c|c|c|c|c|}
\hline \multirow{2}{*}{$\begin{array}{l}\text { Remuneração em } \\
\text { Salários Mínimos }\end{array}$} & \multicolumn{2}{|c|}{2013} & \multicolumn{2}{|c|}{2014} & \multicolumn{2}{|c|}{2015} & \multicolumn{2}{|c|}{2016} & \multicolumn{2}{|c|}{2017} \\
\hline & V.Abs. & $\%$ & V.Abs. & $\%$ & V.Abs. & $\%$ & V.Abs. & $\%$ & V.Abs. & $\%$ \\
\hline Até 0,50 & 124 & 0,41 & 186 & 0,62 & 125 & 0,40 & 187 & 0,59 & 99 & 0,31 \\
\hline 0,51 a 1,00 & 1.661 & 5,53 & 1.380 & 4,58 & 1.442 & 4,66 & 1.620 & 5,15 & 1.603 & 4,96 \\
\hline 1,01 a 1,50 & 15.682 & 52,18 & 15.292 & 50,76 & 15.986 & 51,68 & 16.884 & 53,65 & 16.971 & 52,56 \\
\hline 1,51 a 2,00 & 6.497 & 21,62 & 7.512 & 24,93 & 7.547 & 24,40 & 7.652 & 24,32 & 8.297 & 25,70 \\
\hline 2,01 a 3,00 & 3.736 & 12,43 & 3.723 & 12,36 & 3.466 & 11,21 & 3.230 & 10,26 & 3.152 & 9,76 \\
\hline 3,01 a 4,00 & 555 & 1,85 & 614 & 2,04 & 583 & 1,88 & 550 & 1,75 & 517 & 1,60 \\
\hline 4,01 a 5,00 & 217 & 0,72 & 202 & 0,67 & 315 & 1,02 & 214 & 0,68 & 358 & 1,11 \\
\hline 5,01 a 7,00 & 629 & 2,09 & 540 & 1,79 & 766 & 2,48 & 522 & 1,66 & 603 & 1,87 \\
\hline 7,01 a 10,00 & 272 & 0,91 & 275 & 0,91 & 258 & 0,83 & 149 & 0,47 & 187 & 0,58 \\
\hline 10,01 a 15,00 & 90 & 0,30 & 93 & 0,31 & 35 & 0,11 & 34 & 0,11 & 31 & 0,10 \\
\hline 15,01 a 20,00 & 13 & 0,04 & 7 & 0,02 & 2 & 0,01 & 3 & 0,01 & 4 & 0,01 \\
\hline Mais de 20,00 & 12 & 0,04 & 4 & 0,01 & 9 & 0,03 & 10 & 0,03 & 6 & 0,02 \\
\hline Não classificado & 566 & 1,88 & 300 & 1,00 & 398 & 1,29 & 414 & 1,32 & 460 & 1,42 \\
\hline Total & 30.054 & 100,00 & 30.128 & 100,00 & 30.932 & 100,00 & 31.469 & 100,00 & 32.288 & 100,00 \\
\hline
\end{tabular}

Fonte: Elaborado a partir da RAIS-MTE-2018.

nas uma classe acima do valor mais encontrado. Esse achado sugere as fracas expectativas salariais da categoria.

\section{Dimensão social}

De acordo com a PPEB (2013), 28,3\% dos auxiliares e técnicos de enfermagem sentem-se pro- tegidos contra violência no trabalho. A maioria dos entrevistados afirma que não se sente protegido $(41,7 \%)$ ou que se sente protegido apenas às vezes $(20,0 \%)$.

Esses resultados são coerentes com o relato de existência de violência no trabalho, pois $25,5 \%$ dos entrevistados afirmaram ter sofrido algum tipo de violência no trabalho. Sobre os tipos de 
violência sofrida, predominam a psicológica $(78,4 \%)$, a institucional $(14,2 \%)$ e física $(7,5 \%)$. Destaca-se que nenhum profissional informou haver sofrido violência sexual.

O questionário da PPEB investigou a existência de discriminação no ambiente de trabalho e 20,1\% dos entrevistados responderam positivo essa questão. A discriminação mais relatada é a de gênero $(48,8 \%)$, racial $(21,3 \%)$, peso/obesidade $(21,3 \%)$, orientação sexual $(7,5 \%)$ e pessoa com deficiência (1,3\%).

Quanto à ocorrência de acidente de trabalho nos últimos 12 meses (ano base 2013), identificou-se $16 \%$ do setor privado, $10,4 \%$ do setor filantrópico e $8,8 \%$ do setor público. Sobre a necessidade de licença médica, nesse mesmo período, os dados mostraram o seguinte perfil: $16 \%$ do setor público, $13,6 \%$ do setor privado e $6,3 \%$ do setor filantrópico.

\section{Dimensão organizacional}

Embora os resultados da $\mathrm{PPEB}$ não apresentem dados específicos sobre essa dimensão que discorre sobre a ausência de controle individual ou coletivo das condições de trabalho, a análise considerou discutir esse aspecto em associação com as demais dimensões, por entender que todas estão imbricadas, de modo a fazer interseção em diversos pontos da vida laboral e pessoal dos auxiliares e técnicos de Enfermagem. A diferença entre os ambientes de trabalho dos setores que envolvem essa categoria pode representar ausência de controle individual ou coletivo sobre essas condições.

Entre os profissionais que trabalham no setor público (27.727), as condições de trabalho foram consideradas boas $(35,3 \%)$ e regulares $(35,0 \%)$ pela maioria dos respondentes $(70,3 \%)$; no setor privado (9.186), as respostas privilegiaram as condições boa $(40,7 \%)$ e ótima $(34,3 \%)$, total de $75,0 \%$. O setor filantrópico abrange 4.677 profissionais e as repostas concentraram-se entre as condições regular $(50,9 \%)$ e boa $(34,5 \%)$, considerado o setor que evidencia o menor percentual de respostas atribuindo as condições de trabalho como excelentes $(3,6 \%)$, quando comparados ao privado $(11,1 \%)$ e ao público $(6,1 \%)$.

Em relação as péssimas condições de trabalho, os respondentes sinalizaram, em escala crescente, o setor privado $(0,9 \%)$, o filantrópico $(3,6 \%)$ e o público $(7,1 \%)$.

\section{Discussão}

As condições de trabalho dos profissionais de saúde passaram por densas mudanças resultado das transformações globais ocorridas nos âmbitos ocupacional e de saúde nas últimas décadas ${ }^{22}$, o que desafia governos e sociedade civil ofertar serviços com qualidade nos sistemas de saúde ${ }^{22,23}$.

No Brasil, o SUS tornou-se o principal mercado de trabalho dos profissionais de saúde, aumentando, sobremaneira, os postos de trabalho da Enfermagem na rede pública, sobretudo nos municípios e na rede privada, por meio de contratos ${ }^{24}$ que não suprem as necessidades de o profissional usufruir de uma vida saudável e digna.

Quanto às dimensões econômica e temporal, ainda que a PPEB aponte um quadro positivo quanto a natureza da atividade profissional de auxiliares e técnicos de enfermagem com a "empregabilidade" no serviço público, estes profissionais detêm frágeis vínculos contratuais que não asseguram estabilidade e nem certificam a condição de o profissional ser regido por estatuto do servidor público. A ausência de concursos públicos reforça esse cenário de fragilidade de vínculos contratuais nesse mercado de trabalho.

Em decorrência de múltiplos vínculos empregatícios, com atividade média de 1,35 por profissional, auxiliar ou técnico de enfermagem, exacerbam-se as repercussões na qualidade de vida, como também pode favorecer as ocorrências do erro humano que, na maioria das vezes, acabam por ser imputados à responsabilidade desses profissionais.

Entre os auxiliares e técnicos de enfermagem recaem altos índices de estresses, síndrome de Burnout, adoecimento físico e mental, absenteísmo e insatisfação com as condições de trabalho, além do não reconhecimento social ${ }^{22,23}$.

O aumento da demanda por serviços de saúde, sobrecarga de trabalho e alterações na rotina, interação intensa e contínua com pacientes em sofrimento, pressão por cuidado, escassez de pessoal, déficit de tempo para realizar as tarefas, rotatividade de profissionais, baixos salários, indícios de saturação do mercado de trabalho, fragilidade de vínculos laborais, entre outros, levaram à uma diminuição na qualidade do serviço ofertado, ao aumento de erros e ao esgotamento do profissional, expressado pela exaustão emocional, intelectual e física ${ }^{22-24}$.

Os resultados da PPEB evidenciam alta rotatividade de auxiliares e técnicos de Enfermagem nos locais de trabalho em busca de melhorias salariais, de melhores condições de trabalho e a 
imperiosa necessidade de conciliar múltiplos vínculos. A sobrecarga de trabalho com carga-horária semanal é desumana e incompatível com um cuidado atento e responsável. Identifica-se que não é incomum, profissionais que trabalhem mais de 60 horas semanais. Nesse sentido, a reforma trabalhista proposta em 2016 pelo governo brasileiro, que prevê aumento da jornada de trabalho e predomínio do negociado sobre o legislado, limitando a atuação governamental nas questões trabalhistas ${ }^{25}$, pode agravar e chancelar a sobrecarga da jornada excessiva da categoria.

No cenário cearense, a perspectiva da precarização do trabalho amplia-se na medida em que o rendimento mensal auferido, somando os múltiplos vínculos, pela maioria desses profissionais, situa-se entre um a três salários mínimos, correspondendo, na época da pesquisa, entre $\mathrm{R} \$ 681,00$ a 2.000,00 reais. Esse cenário de baixos salários e dificuldades de manutenção do emprego, configuram precarização do trabalho, segundo as dimensões temporal e econômica sob a ótica da OIT $^{19}$.

O setor público estadual e municipal absorve maior quantidade de profissionais, embora ascenda a modalidade de cooperativas como panorama de empregabilidade nesta categoria, não assegura os direitos trabalhistas, amplia o fosso na busca de condições dignas de trabalho e revela fragilidade organizacional e social das dimensões trabalhistas.

É necessário considerar, de modo adicional, que a flexibilização das relações de trabalho ocorre por meio de contratações atípicas, terceirizadas, sem concurso público, perpetuando uma política que transforma a natureza do Estado e de sua intervenção ${ }^{16}$.

Neste estudo, atenta-se para o elevado percentual de profissionais cooperativados no Ceará, o que confirma a precarização dos vínculos trabalhistas que podem desencadear sérias repercussões na segurança do paciente, comprometendo assim, a qualidade da atenção, conforme apresentado na PPEB.

Destaca-se a adoção preferencial pelos gestores municipais de saúde quanto à tipos 'flexíveis' de contratos de trabalho; o regime de terceirização para cooperativas e empresas; os contratos de autônomos; além de outros tipos que conformam exploração da força de trabalho em saúde. Este fato pode ser observado em muitos municípios brasileiros na Atenção Primária à Saúde (APS), especialmente na Estratégia Saúde da Família $(\mathrm{ESF})^{17}$.

Outro resultado expressivo é a diferença de respostas de acordo com os locais de trabalho. No caso dos acidentes de trabalho, no setor privado exibe o dobro de eventos em relação ao público. No caso das licenças médicas, o setor público expressa quase o triplo do setor filantrópico. Não é possível, segundo os dados da PPEB, estabelecer um padrão claro sobre onde estão as piores e melhores condições de trabalho.

Alves $^{26}$ advoga que a precarização resulta das relações instáveis do trabalho, das relações flexíveis e informais laborais, as quais provocam a rotatividade elevada e a ausência de benefícios ou salários indiretos, a insegurança do vínculo, baixa remuneração, largas jornadas de trabalho, dentre outros prejuízos ao trabalhador.

Nesta definição, também são consideradas as condições inadequadas de trabalho e as contratações temporárias que denotam o sentimento de instabilidade causada ao trabalhador. O caráter de precariedade remete não apenas à eventual redução da proteção social ao trabalho, o que implica em menor número de direitos e benefícios, mas ao fato desse tipo de contrato ter duração curta ou delimitada no tempo, proporcionando um sentimento de instabilidade ${ }^{16,27}$.

Os tipos de contratos de trabalhos em cenários tencionados e impulsionados por políticas neoliberais, em detrimentos das sociais, como no Brasil, aumentam a insegurança da força de trabalho no setor saúde e afins, levando órgãos nacionais e internacionais a convocarem esforços para coibirem as situações que se assemelham ao trabalho escravo.

Nesse sentido, o termo 'flexibilização' referese às várias formas de modificação do direito trabalhista, que compatibilizam mudanças de ordem econômica, tecnológica, política e social existentes na relação entre o capital e o trabalho. Esse fenômeno decorre de fatores, dentre estes, tecnologias, desemprego, globalização e, principalmente, a crise econômica ${ }^{28}$, os quais atingem, sobremaneira, as profissões que ainda não galgaram remunerações dignas e reconhecimento social.

Mostra-se tímido o olhar para a ampliação no campo de trabalho nessa categoria como autônomo e prestando serviços na modalidade de homecare. A transição demográfica no cenário global e local, descortina uma área de atuação promissora para a categoria, com possível ampliação de renda, satisfação no trabalho, reconhecimento familiar e social.

Estudos internacionais apóiam essa compreensão, ao afirmar que na medida em que a população envelhece, o número de pessoas que necessita de homecare aumenta e essa assistência em domicílio tem acrescentado mais empregos à 
economia do que qualquer outra ocupação iso$\operatorname{lada}^{29,30}$.

No contexto global, a longevidade implica no aumento das condições crônicas e compromete a sustentabilidade dos sistemas de saúde. Embora o quantitativo de auxiliares e técnicos de Enfermagem que atuam no homecare seja baixo $(0,5 \%)^{3}$, esse campo de prestação de serviços traz outros benefícios, como a diminuição dos riscos de infecção em ambientes hospitalares, favorece o fortalecimento das relações interpessoais no ambiente domiciliar, reduz complicações clínicas e otimiza o tempo de recuperação do paciente.

No entanto, este cenário demandará, cada vez mais, uma formação qualificada e alinhada com as inovações tecnológicas que incrementam as maneiras de cuidar e gerenciar o cuidado em domicílio, em Instituições de Longa Permanência para a Pessoa Idosa, Creches Infantis e outros espaços que abriguem populações demandantes desse cuidado.

Em contraponto, o perfil dessa categoria está em transição educacional, ampliando status e conquistas, mas com nível interpretativo-analítico que carece adequar-se às exigências de conhecimento multidimensional, centrado em valores humanos, éticos e legais.

De fato, conectar diferentes conhecimentos e habilidades é considerado uma característica importante do trabalho de Enfermagem domiciliar. A transferência do cuidado institucional para o domiciliar é uma das maiores mudanças nos cuidados de saúde modernos, acrescentando que pacientes e familiares tornaram-se parte importante da "rede de cuidados"30.

A prática de enfermagem em domicílio é em grande parte relacional, capturada na ideia de criar uma rede em torno de cada paciente para garantir a assistência necessária para que as pessoas possam permanecer em suas casas. É preciso introduzir conhecimentos e habilidades relacionadas ao trabalho em equipe, organização de cuidados e questões éticas embutidas na compreensão do bom atendimento e gerenciamento das ações de cuidado praticadas pela equipe de Enfermagem $^{30}$.

Ao se analisar a responsabilidade de lidar com vidas, primar pela não ocorrência de erro humano e, ao mesmo tempo, perceber-se incapaz de manter a dignidade de sua vida e de familiares, infere-se a imersão dessa categoria em sensação de desesperança. Esta pode ser potencializada pela exaustão física decorrente, dentre outros motivos, de privação de sono, além de períodos incompatíveis com o descanso físico e mental.

A sensação de segurança contra violência nos locais de trabalho foi negada, de algum modo, pela maioria $(61,7 \%)$, atestando os elevados índices de violência que colocam o estado do Ceará como um dos mais violentos do país ${ }^{31}$, evidenciando debilidade nos aspectos organizativos das instituições e de segurança social.

Importante ressaltar que ao discorrerem sobre terem sofrido algum tempo de violência, não houve menção a violência sexual entre os respondentes. É possível ter havido receio em verbalizarem sobre violência sexual, criminalizada por lei, pois poderia incorrer em tomada de decisão que implicasse retaliações nos vínculos de trabalho, ou perda do emprego.

Em contraponto, um percentual dos participantes $(20,1 \%)$ confirmou a existência de discriminações no ambiente de trabalho e, dentre estas, prevaleceu a discriminação de gênero e de raça, categorias que apresentam fortes ligações com as ocorrências das manifestações de violência sexual ${ }^{7}$.

Pode-se deduzir que há um viés quando se relaciona a resposta totalmente negativa acerca da 'violência sexual' e aqueles respondentes que afirmaram ter sofrido 'discriminação de gênero', o que leva a duas possibilidades: o entendimento dos participantes acerca do que seria violência sexual no instrumento de coleta, o qual pode estar relacionado estritamente ao ato do estupro no ambiente de trabalho; ou a hipótese da resposta negativa mediante questões culturais relacionadas à estigma e preconceito.

\section{Conclusão}

A discussão crítica dos resultados da PPEB para o Ceará evidencia que a precarização do trabalho de auxiliares e técnicos de enfermagem é sustentada por amplas evidências empíricas. Os dados da RAIS corroboram os achados da PPEB e apontam que inexiste qualquer tendência de melhora dessa situação. A principal limitação desse estudo é basear-se em dados secundários, não sendo possível aprofundar a compreensão sobre a dimensão organizacional da precarização do trabalho. Seria relevante a realização de pesquisa empírica para compreender as implicações da relação entre profissionais e instituições na precarização do trabalho. 


\section{Colaboradores}

RM Silva e LJES Vieira participaram na concepção, análise e interpretação dos dados, redação e revisão final do artigo; C Garcia Filho, IC Bezerra, AN Cavalcante, FC Borba Netto e FAR Aguiar trabalharam na coleta, análise e interpretação dos dados, redação do artigo.

\section{Referências}

1. Machado MH, Wermelinger M, Vieira M, Oliveira ES, Lemos WR, Filho W, Lacerda WF, Santos M, Junior P, Justino E, Barbosa, C. Aspectos gerais da formação da enfermagem: o perfil da formação dos enfermeiros técnicos e auxiliares. Enfermagem em Foco 2016; 7(n. esp.).15-34.

2. Machado MH, Oliveira ES, Lemos WR, Lacerda WF, Justino E. Mercado de trabalho em enfermagem no âmbito do SUS: uma abordagem a partir da pesquisa Perfil da enfermagem no Brasil. Divulgação em Saúde para Debate 2016; 56:98-105.

3. Machado MH, Oliveira ES, Lemos WR, Lacerda WF, Aguiar Filho W, Wermelinger M, Vieira M, Santos M, Souza Junior PB, Justino E, Barbosa C. Mercado de trabalho da enfermagem: aspectos gerais. Enfermagem em Foco 2016; 7(n. esp.):35-53.

4. Persegona MFM, Oliveira ES, Pantoja VJC. As características geopolíticas da enfermagem brasileira. Divulgação em Saúde para Debate 2016; 56:19-35.

5. Walker F. An examination of a nursing assistant role in an acute hospital setting [thesis]. Melbourne: RMIT University; 2019.

6. Conselho Federal de Enfermagem (Cofen). Resolução Cofen no 418/2011. Atualiza, no âmbito do sistema Cofen/Conselhos Regionais de Enfermagem, os procedimentos para registro de especialização técnica de nível médio em Enfermagem. Diário Oficial da União 2011; 6 dez.

7. Conselho Federal de Enfermagem (Cofen). Pesquisa Perfil da Enfermagem no Brasil. Banco de Dados. Relatório Final. [internet]. [acessado 2019 Abr 15]. Disponível em: http://www.cofen.gov.br/perfilenfermagem/

8. Ribeiro GT. Sistematização da assistência de enfermagem: aspecto substantivo da prática profissional. Escola Anna Nery Revista de Enfermagem 2016; 20(1):5-6.

9. Benedet S, Gelbcke F, Amante L, Padilha M, Pires D. Processo de Enfermagem: instrumento da Sistematização da Assistência de enfermagem na percepção dos enfermeiros. Revista de Pesquisa: Cuidado é Fundamental Online 2016; 8(3):4780-4788.

10. Soares M, Rodrigues RZ, Camelo S, Souza TF. Gestión de recursos humanos y su interfaz en la sistematización de la asistencia de enfermería. Enfermería Global 2016; 15(2):341-375.

11. Santos W, Santos AM, Lopes, TRP, Madeira, MZ, Rocha FC. (). Sistematização da Assistência de Enfermagem: o contexto histórico, o processo e obstáculos da implantação. JMPHC 2014; 5(2):153-158.

12. Gutiérrez MGR, Morais SCRV. Systematization of nursing care and the formation of professional identity. Rev Bras Enferm 2017;70(2):436-441.

13. Zavala MOQ, Klinj TP, Carrillo KLS. Quality of life in the workplace for nursing staff at public healthcare institutions. Rev. Latino-Am. Enfermagem [Internet]. 2016 [acessado 2019 Abr 25]; 24:e2713. Disponível em: http://www.scielo.br/scielo.php?script=sci_arttext \&pid=S0104-11692016000100355\&lng=en 
14. Miorin JD, Camponogara S, Pinno C, Beck CLC, Costa V, Freitas EO. Prazer e sofrimento de trabalhadores de enfermagem de um pronto-socorro. Texto contexto - enferm. [Internet]. 2018 [acessado 2019 Abr 25]; 27(2):e2350015. Disponível em: http:// www.scielo.br/scielo.php?script $=$ sci_arttext\&pi$\mathrm{d}=$ S0104-07072018000200305\&lng=en

15. Vieira GLC. Agressão física contra técnicos de enfermagem em hospitais psiquiátricos. Rev. bras. saúde ocup. [Internet]. 2017 [acessado 2019 Abr 25]; 42:e8. Disponível em: http://www.scielo.br/scielo.php?script=sci_arttext\&pid=S0303-76572017000100206\&lng=en

16. Druck G. A terceirização na saúde pública: formas diversas de precarização do trabalho. Trabalho, Educação e Saúde 2016; 14(Supl.1):15-43.

17. Schimith MD, Brêtas ACP, Simon BS, Brum DJT, Alberti GF, Bidó MLD, Gomes TF. Precarização e fragmentação do trabalho na estratégia saúde da família: impactos em santa maria (RS). Trabalho, Educação e Saúde 2017; 15(1):163-182.

18. Rizzotto MLF. Editorial. Divulgação em Saúde para Debate 2016; 56:4-5.

19. International Labour Office. From precarious work to decent work: outcome document to the workers' symposium on policies and regulations to combat precarious employment. Geneva: International Labour Office; 2012. [acessado Abr 20]. Disponível em: http://www. ilo.org/wcmsp5/groups/public/@ed_dialogue/@actrav/documents/meetingdocument/wcms_179787. pdf

20. Rodrigues AMU, Matsue RY, Abreu RNDC, Moreira LC, Frota MA. Estratégias defensivas no trabalho dos profissionais da saúde de uma emergência no Nordeste brasileiro. Divulgação em Saúde para Debate 2016; 56:171-178.

21. Instituto Brasileiro de Geografia e Estatística (IBGE). Bases de dados»metadados»MTE»relação anual de informações sociais - RAIS. 2019. [acessado 2019 Abr 20]. Disponível em: https://ces.ibge.gov.br/base-dedados/metadados/mte/relacao-anual-de-informacoes-sociais-rais.html

22. Nogueira LS, Sousa RMC, Guedes ES, Santos MA, Turrini RNT, Cruz DALM. Burnout e ambiente de trabalho de enfermeiros em instituições públicas de saúde. Rev. Bras. Enferm. [Internet]. 2018 Apr [acessado 2019 Fev 21]; 71(2):336-342. Disponível em: http://www.scielo.br/scielo.php?script=sci_arttext\&pid=S0034-71672018000200336\&lng=en. http:// dx.doi.org/10.1590/0034-7167-2016-0524.

23. Mudallal RH, Saleh MYN, Al-Modallal HM, Abde1-Rahman RY. Quality of Nursing Care: The Influence of Work Conditions and Burnout. International Journal of Africa Nursing Sciences. [Internet] 2017 [acessado 2019 Abr 22]; 7:24-30. Disponível em: https:// www.researchgate.net/publication/317693344_Quality_of_Nursing_Care_The_Influence_of_Work_Conditions_and_Burnout
24. Freire NPF. Trajetória: Fatos e Fotos da Pesquisa Perfil da Enfermagem. Enferm. Foco. 2016; 7(n. esp.):8186. [online]. [acessado $2019 \mathrm{Fev} 21$ ]. Disponível em: http://revista.cofen.gov.br/index.php/enfermagem/ article/viewFile/701/310

25. Santos RR, Lima EFA, Freitas PSS, Galavote HS, Rocha EMS, Lima RCD. A influência do trabalho em equipe na Atenção Primária à Saúde. Rev. Bras. Pesq. Saúde 2016; 18(1):130-139.

26. Alves G. Neodesenvolvimentismo e precarização do trabalho no Brasil (2003-2013). Cadernos de Ciências Sociais (Porto de Ideias) 2015; 4:81-94.

27. Eberhardt LD, Carvalho M, Murofuse NT. Vínculos de trabalho no setor saúde: o cenário da precarização na macrorregião Oeste do Paraná. Saúde em Debate [online] 2015 [acessado $2019 \mathrm{Fev} 25$ ]; 39(104):1829. Disponível em: https://doi.org/10.1590/0103110420151040432

28. Parreira ASM, Rezende CMC, Borges LH, Mendonça CCA, Massaroni L, Ambrósio M PM. A flexibilização das relações de trabalho na saúde: a realidade de um Hospital Universitário Federal. Cien Saude Colet [Internet]. 2015 Oct [acessado 2019 Feb 25];20(10):3043-3050. Disponível em: http:// search.ebscohost.com $/ \operatorname{login}$. aspx?direct $=$ true $\& \mathrm{db}=$ foh\&AN=110341746\&lang=pt-br\&site $=$ eds-live

29. Butler SS. Exploring Relationships Among Occupational Safety, Job Turnover, and Age Among Home Care Aides in Maine. New Solutions: A Journal of Environmental and Occupational Health Policy 2018; 27(4):501-523.

30. Bjornsdottir K. I try to make a net around each patient': home care nursing as relational practice. Scand J Caring Sci 2018; 32:177-185.

31. Waiselfisz JJ. Mapa da violência 2016. Homicídios por Arma de Fogo no Brasil. São Paulo: Flacso Brasil; 2016. [acessado 2019 Abr 25]. Disponível em: https://www. mapadaviolencia.org.br/pdf2016/Mapa2016_armas_ web.pdf

Artigo apresentado em 29/04/2019

Aprovado em 20/08/2019

Versão final apresentada em 03/10/2019 
